\title{
The Hidden Mass and Large Spatial Extent of a Poststarburst Galaxy Outflow
}

\author{
Todd M. Tripp, ${ }^{1 *}$ Joseph D. Meiring, ${ }^{1}$ J. Xavier Prochaska, ${ }^{2}$ \\ Christopher N. A. Willmer, ${ }^{3}$ J. Christopher Howk, ${ }^{4}$ Jessica K. Werk, ${ }^{2}$ \\ Edward B. Jenkins, ${ }^{5}$ David V. Bowen, ${ }^{5}$ Nicolas Lehner, ${ }^{4}$ \\ Kenneth R. Sembach, ${ }^{6}$ Christopher Thom, ${ }^{6}$ and Jason Tumlinson ${ }^{6}$ \\ ${ }^{1}$ Department of Astronomy, University of Massachusetts, Amherst, MA 01003, USA \\ ${ }^{2}$ UCO/Lick Observatory, University of California, Santa Cruz, CA 95064, USA \\ ${ }^{3}$ Steward Observatory, University of Arizona, Tucson, AZ 85721, USA \\ ${ }^{4}$ Department of Physics, University of Notre Dame, Notre Dame, IN 46556, USA \\ ${ }^{5}$ Princeton University Observatory, Princeton, NJ 08544, USA \\ ${ }^{6}$ Space Telescope Science Institute, Baltimore, MD 21218, USA \\ *To whom correspondence should be addressed; E-mail: tripp@ astro.umass.edu.
}

Outflowing winds of multiphase plasma have been proposed to regulate the buildup of galaxies, but key aspects of these outflows have not been probed with observations. Using ultraviolet absorption spectroscopy, we show that "warm-hot" plasma at $10^{5.5} \mathrm{~K}$ contains 10-150 times more mass than the cold gas in a poststarburst galaxy wind. This wind extends to distances $>68$ kiloparsecs, and at least some portion of it will escape. Moreover, the kinematical correlation of the cold and warm-hot phases indicates that the warm-hot plasma is related to the interaction of the cold matter with a hotter (unseen) phase at $\gg 10^{6} \mathbf{K}$. Such multiphase winds can remove substantial masses and alter the evolution of poststarburst galaxies.

Galaxies do not evolve in isolation. They interact with other galaxies and, more subtly, with the gas in their immediate environments. Mergers of comparable-mass, gas-rich galaxies trigger star-formation bursts by driving matter into galaxy centers, but theory predicts that such starbursts are short-lived: the central gas is rapidly driven away by escaping galactic winds powered by massive stars and supernova explosions or by a central supermassive black hole (1). 
Such feedback mechanisms could transform gas-rich spiral galaxies into poststarburst galaxies (2) and eventually into elliptical-type galaxies with little or no star formation (3). Mergers are not required to propel galaxy evolution, however. Even relatively secluded galaxies accrete matter from the intergalactic medium (IGM), form stars, and drive matter outflows into their halos or out of the galaxies entirely $(4,5)$. In either case, the competing processes of gas inflows and outflows are expected to regulate galaxy evolution.

Outflows are evident in some nearby objects (6-9) and are ubiquitous in some types of galaxies (10-15); their speeds can exceed the escape velocity. Nevertheless, their broader impact on galaxy evolution is poorly understood. First, their full spatial extent is unknown. Previous studies $(6,9,16-22)$ have revealed flows with spatial extents ranging from a few parsecs up to $\approx 20$ kiloparsecs (kpc). However, because of their low densities, outer regions of outflows may not have been detected with previously used techniques, and thus the flows could be much larger. Second, the total column density and mass of the outflows is poorly constrained. Previous outflow observations are often limited to low-resolution spectra of only one or two ions (e.g., Na I or Mg II) or rely on composite spectra that cannot yield precise column densities. Without any constraints on hydrogen (the vast bulk of the mass) or other elements and ions, these studies are forced to make highly uncertain assumptions to correct for ionization, elemental abundances, and depletion of species by dust. Finally, galactic winds contain multiple phases with a broad range of physical conditions ( 6 ), and wind gas in the key temperature range between $10^{5}-10^{6}$ $\mathrm{K}$ (where radiative cooling is maximized) is too cool to be observed in X-rays; detection of this so-called "warm-hot" phase requires observations in the ultraviolet (UV).

To study the more extended gas around galaxies, including regions affected by outflows, we used the Cosmic Origins Spectrograph (COS) on the Hubble Space Telescope (HST) to obtain high-resolution spectra of the quasistellar object (QSO) PG1206+459 $\left(z_{\mathrm{QSO}}=1.1625\right)$. By exploiting absorption lines imprinted on the QSO spectrum by foreground gaseous material, we can detect the low-density outer gaseous envelopes of galaxies, regions inaccessible to other techniques. We focus on far-ultraviolet (FUV) absorption lines at rest wavelengths $\lambda_{\text {rest }}<912 \AA$. This FUV wavelength range is rich in diagnostic transitions (23), including the Ne VIII 770.409, 780.324 doublet, a robust probe of warm-hot gas, as well as banks of adjacent ionization stages. The sight line to PG1206+459 pierces an absorption system, at redshift $z_{\text {abs }}=0.927$, that provides insights about galactic outflows. This absorber has been studied before (24), but previous observations did not cover Ne VIII and could not provide accurate constraints on $\mathrm{H} \mathrm{I}$ in the individual absorption components.

This absorber is illustrated in Figures $1-3$ including the COS data (25). The absorber is a "partial" Lyman limit (LL) system (i.e., the higher Lyman series lines are not saturated), which enables accurate $N(\mathrm{H} \mathrm{I})$ measurement (Fig. 11). A wide variety of metals and $\mathrm{H}$ I lines are detected in at least 9 components (25) spanning a large velocity range from -317 to $+1131 \mathrm{~km}$ $\mathrm{s}^{-1}$ (Figs. 112). The Ne VIII doublet is unambiguously detected (Fig. 2) with a total Ne VIII column density of $N(\mathrm{Ne}$ VIII $)=10^{14.9} \mathrm{~cm}^{-2}(25)$, which is $\approx 10 \times$ higher than any previous $N\left(\mathrm{Ne}\right.$ VIII) detections in intervening absorbers $(26,27)$. The component at $+1131 \mathrm{~km} \mathrm{~s}^{-1}$ exceeds $v_{\text {escape }}$ of any individual galaxy, and the other components have very similar properties 

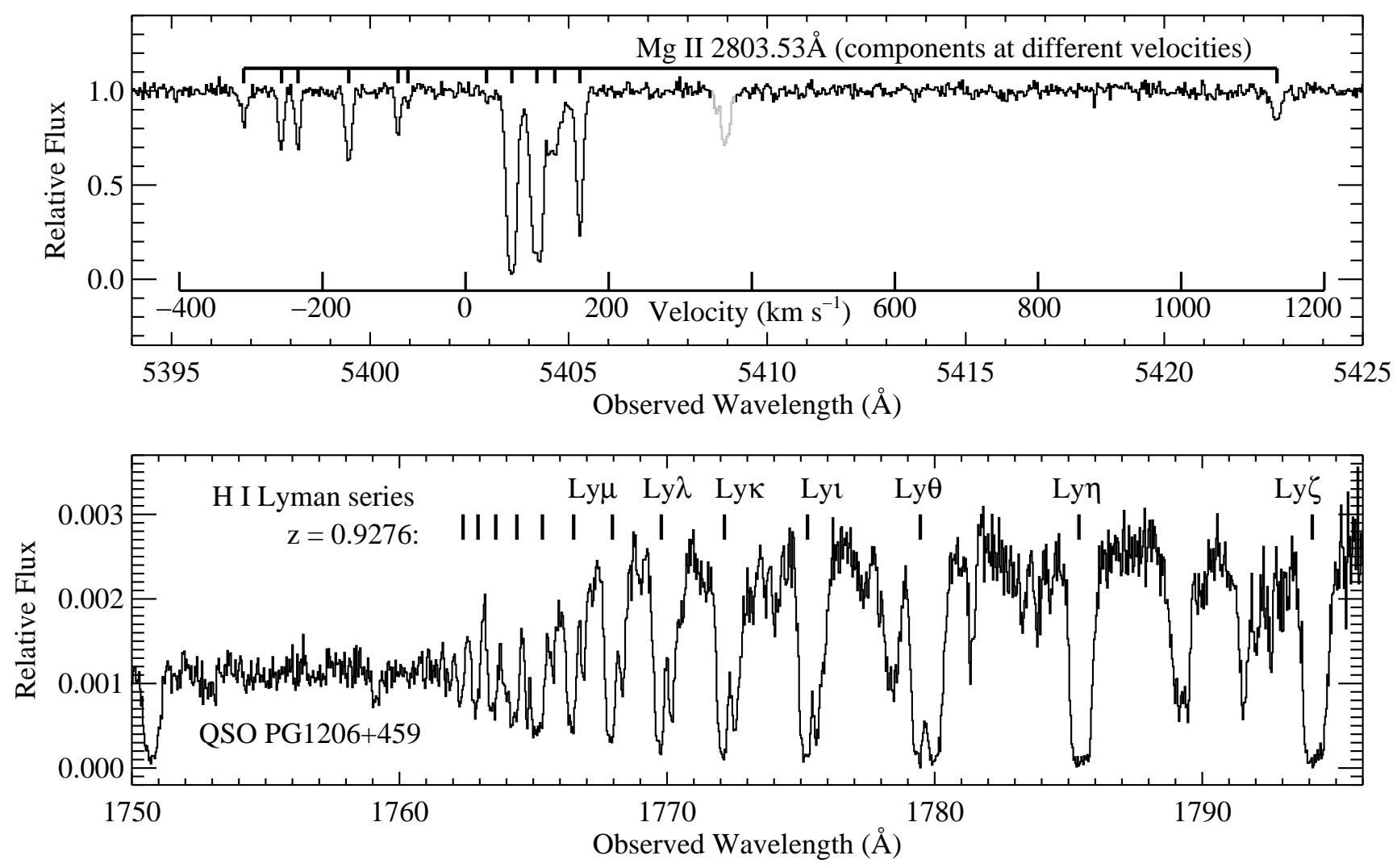

Figure 1: (Top) Small portion of the Keck HIRES spectrum of PG1206+459 (24). Tick marks at top indicate components detected at various velocities in the Mg II $2803.53 \AA$ transition. A velocity scale in the rest frame of the affiliated galaxy 177_9 is inset at bottom. Gray indicates a feature not due to $\mathrm{Mg}$ II 2803.53 $\AA$. (Bottom) Small portion of the ultraviolet spectrum of PG1206+459 recorded with the Cosmic Origins Spectrograph (COS) on HST that shows H I Lyman series absorption lines (marked with ticks and labels) at the redshift of the Mg II complex in the upper panel, including $\mathrm{H} \mathrm{I} \mathrm{Ly} \zeta$ through $\mathrm{Ly} \sigma$ (highest lines are marked but not labeled). 

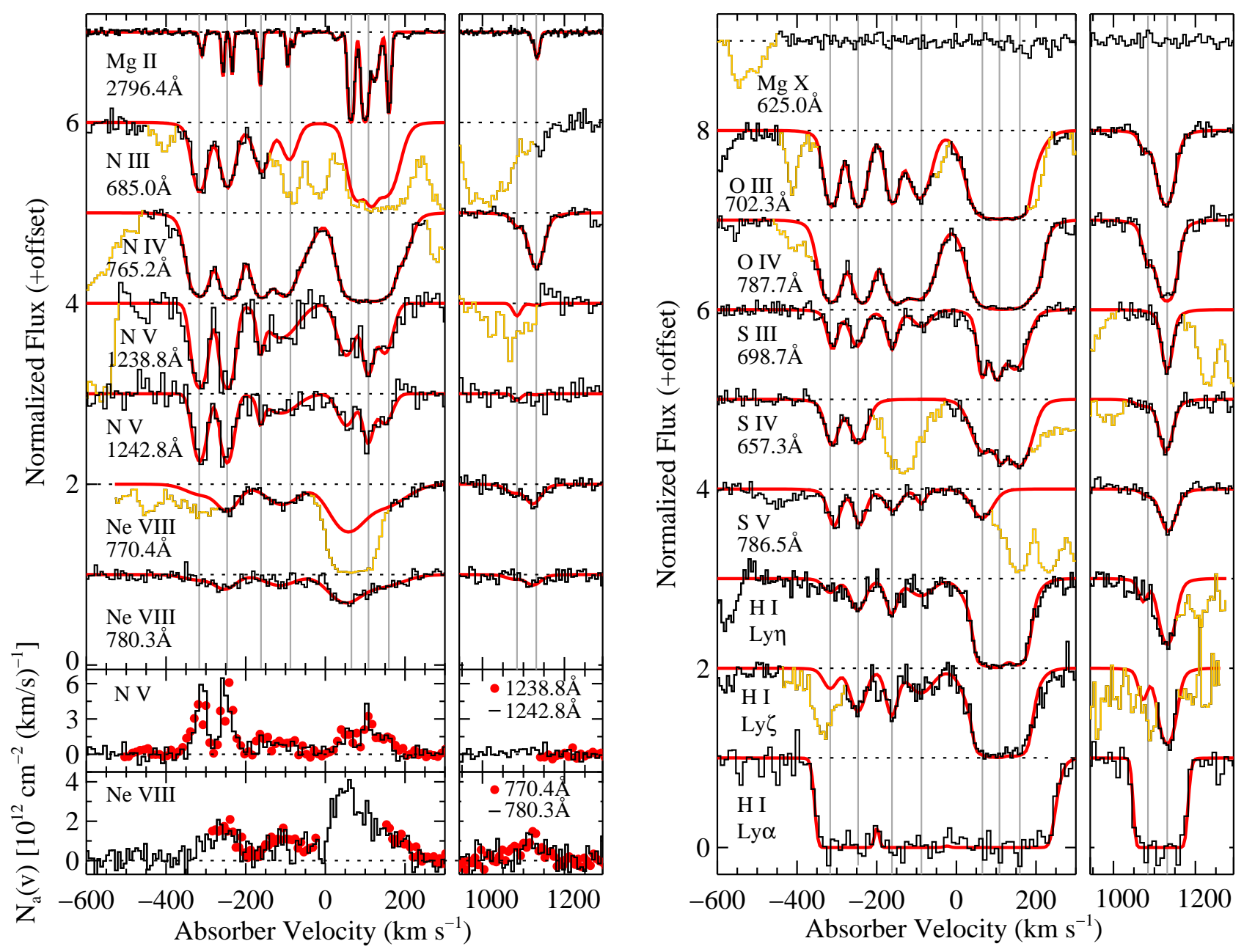

Figure 2: Continuum-normalized absorption profiles (black lines) of various species detected in the Lyman limit/Mg II absorber shown in Figure 1, plotted in velocity with respect to the galaxy 177_9 redshift (i.e., $v=0 \mathrm{~km} \mathrm{~s}^{-1}$ at $z=0.927$ ). Labels below each absorption profile indicate the species and rest wavelength. We fitted 9 components to the COS and STIS data (24). Component centroids are indicated by gray lines, and the Voigt-profile fits are overplotted with red lines (25). Yellow lines indicate contaminating features from other redshifts/transitions. The two panels at lower left compare apparent column density profiles (39) of the $\mathrm{N} V$ and $\mathrm{Ne}$ VIII doublets. 
to the $+1131 \mathrm{~km} \mathrm{~s}^{-1}$ component (25), suggesting a common origin. Whether the other components have $v>v_{\text {escape }}$ depends on the (unknown) potential well, but allowing for projection effects and noting that the gas is already far from the affiliated galaxy (see below), several of the other components could also be escaping. Combined with detection of Ne VIII, the detections of banks of adjacent ions (N II/N III/N IV/N V;O III/O IV;S III/S IV/S V) place tight constraints on physical conditions of the gas. Notably, the velocity centroids and profile shapes of lower and higher ionization stages are quite similar (Figure 3).

This strong Ne VIII/LL absorber is affiliated with a galaxy near the QSO sight line $(24,25)$. This galaxy, which we refer to as 177_9, is the type of galaxy expected to drive a galactic superwind (Fig. 4). Like poststarburst (11) and ultraluminous infrared galaxies (28), galaxy 177_9 is very luminous and blue (29) - based on $\mathrm{M}^{*}$ from DEEP2 (30), the galaxy luminosity $L=1.8 L^{*}$. The MMT spectrum in Fig. 4 is also similar to those of the poststarburst galaxies in (11), with higher Balmer series absorption lines, [O II] emission, and [Ne V] emission indicative of an AGN (25). Most importantly, the galaxy has a large impact parameter from the QSO sight line, $\rho=68 \mathrm{kpc}(31)$, which implies that the gaseous envelope of 177_9 has a large spatial extent.

The component-to-component similarity of the absorption lines (Fig. 3) suggests a related origin. To further investigate the nature of this absorber, we have used photoionization models (32) to derive ionization corrections and elemental abundances (25). These models indicate that the individual components have high abundances ranging from $\approx 0.5 \times$ to $3 \times$ those in the Sun (Table S2). Such high abundances (or metallicities) favor an origin in outflowing ejecta enriched by nucleosynthesis products from stars; at the large impact parameter of 177_9, corotating outerdisk/halo gas or tidal debris from a low-mass satellite galaxy would be expected to have much lower metallicity. Tidal debris from a massive galaxy could have high metallicity, but we are currently aware of only one luminous galaxy near the sight line at the absorber redshift (33); another luminous galaxy interacting with 177_9 is not evident. The absorber could also be intragroup gas, but somehow it must have been metal enriched, so some type of galactic outflow is implicated in any case.

The photoionization models also constrain the total hydrogen column (i.e., $\mathrm{H} \mathrm{I}+\mathrm{H}$ II), and combined with $r \geq 68 \mathrm{kpc}$, this allows mass estimates. Using fiducial thin-shell models (25), we find that the mass of cool, photoionized gas in individual components ranges from $0.6 \times 10^{8}-14 \times 10^{8}$ solar masses. However, photoionization fails (sometimes by orders of magnitude) to produce enough $\mathrm{S} \mathrm{V}, \mathrm{N} \mathrm{V}$, and Ne VIII; these species must arise in hot gas at $T>10^{5} \mathrm{~K}$. Using equilibrium and non-equilibrium collision ionization models (25), we find that the warm-hot gas contains much more mass than the cold gas, with individual components harboring $10 \times 10^{8}-400 \times 10^{8} M_{\odot}$ in hot material. These are rough estimates with many uncertainties. For example, if the absorption arises in thin filaments analogous to those seen in starburst galaxies ( 6 ) or AGN bubbles (34), the cold-gas mass could reduce to $\approx 10^{6} M_{\odot}$ per component. However, as in the thin-shell models, the warm-hot gas could harbor 10-150 $\times$ more mass in such filaments (25). In either case (shells or filaments), given the similarity of the cold and warm-hot absorption lines (Fig. 3), the Ne VIII-bearing plasma must be a transitional phase that links the colder and hotter material and thus provides insights on the outflow physics. The 


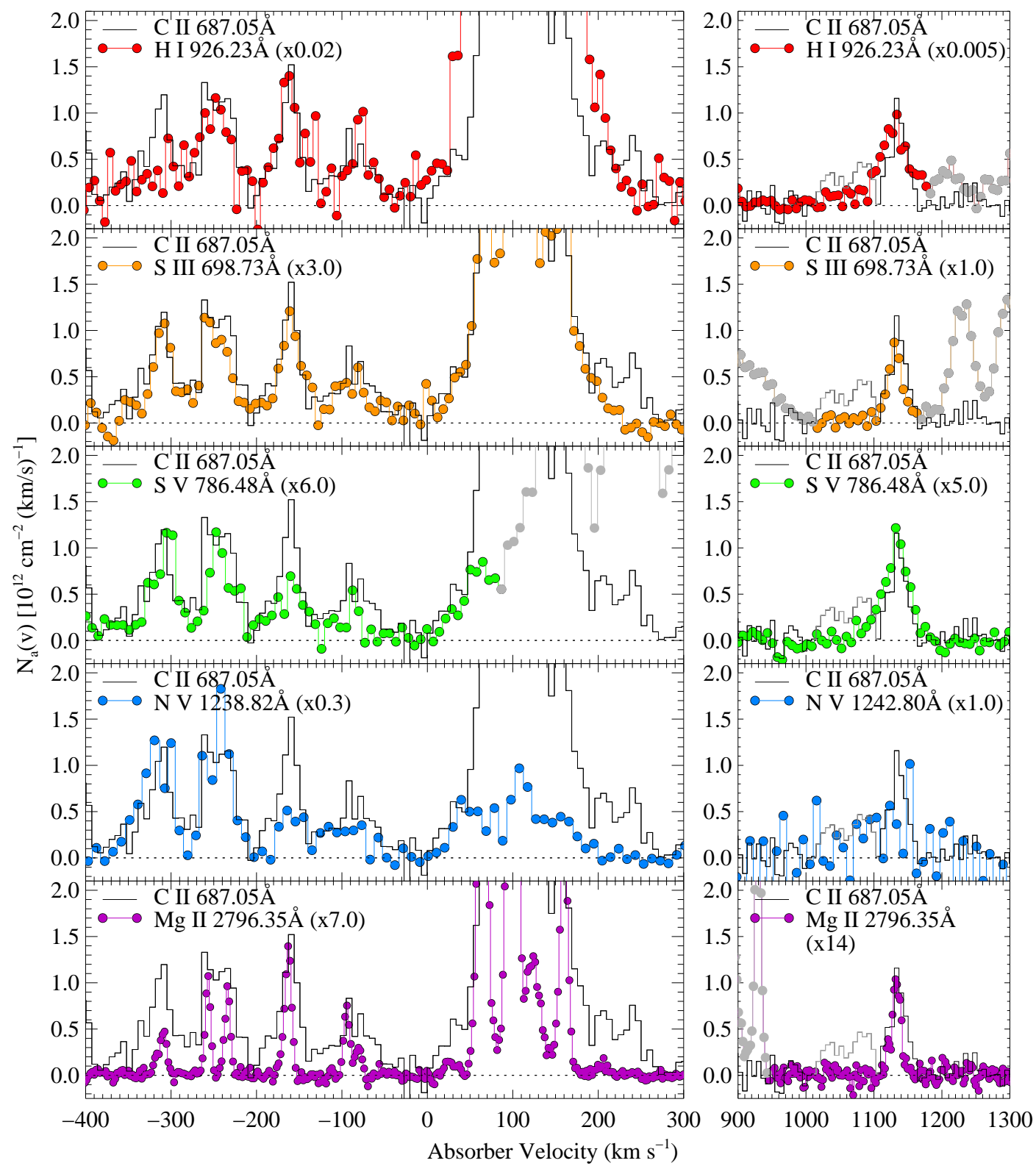

Figure 3: Comparison of apparent column density profiles (39) of the Lyman limit absorber affiliated with galaxy 177_9. In each panel, the C II $687.05 \AA$ profile (black histogram) is compared to another species (colored circles) as labeled at upper left; the comparison species profile is also scaled by the factor in parentheses following the species label. Gray lines indicate regions contaminated by unrelated absorption. As in Figure $2, v=0 \mathrm{~km} \mathrm{~s}^{-1}$ at $z=0.927$. 
$\mathrm{Ne}$ VIII/N v phase is not photoionized, so it must be generated through interaction of the cold gas with a hotter ambient medium analogous to X-ray emitting regions seen in nearby galaxies. How this occurs is an open question; the absorbers could be material cooling from the hot phase down to the cool gas, or the cool clouds could have a hotter and more ionized surface that is evaporating.

Low-density plasma in the $T=10^{5}-10^{6} \mathrm{~K}$ range has been effectively hidden from most outflow studies. In principle, the $\mathrm{O}$ VI 1032,1038 $\AA$ doublet can reveal such gas, but it is unclear whether the $\mathrm{O}$ VI arises in photoionized $10^{4} \mathrm{~K}$ gas, hotter material at $\approx 10^{5.5} \mathrm{~K}$, or both (35). The Ne VIII doublet avoids this ambiguity, and we have found that this warm-hot matter is a substantial component in the mass inventory of a galactic wind. Moreover, this wind has a large spatial extent, and the mass carried away by the outflow will affect the evolution of the galaxy. While earlier studies of poststarburst outflows focused on Mg II and could not precisely constrain the metallicity, hydrogen column, and mass, these studies do indicate that poststarburst outflows are common: 22/35 of the poststarbursts in (36) showed outflowing Mg II absorption with maximum (radial) velocities of $500-2400 \mathrm{~km} \mathrm{~s}^{-1}$, similar to the absorption near 177_9 (Figure 1), and 77\% and 100\% of the poststarburst and AGN galaxies, respectively, in (37) drive outflows but with lower maximum velocities, which may be due to selection of wind-driving galaxies in a later evolutionary stage. With existing COS data, the effects of largescale outflows on galaxy evolution can be studied with the techniques presented here but with larger samples (38), with which it will be possible to statistically track how outflows affect galaxies.

\section{Supporting Online Material:}

Figs. S1 to S5

Tables $\mathrm{S} 1$ to $\mathrm{S} 2$

References (40-54)

Data reduction and column density measurements. We have reduced the Cosmic Origins Spectrograph observations of PG1206+459 as described in (40), including careful alignment of individual exposures (which are intentionally offset on the detector to reduce the effects of fixed-pattern noise), flatfielding, and direct determination of uncertainties from the source and background counts (COS has a photon-counting detector). The default COS data are highly oversampled, so we binned the spectra to Nyquist sampling of the $15 \mathrm{~km} \mathrm{~s}^{-1}$ spectral resolution of the instrument.

Our column-density measurements are summarized in Table S1. A unique aspect of the HST/COS data presented in this paper is the coverage of lines in the rest-frame far-ultraviolet (FUV) at wavelengths between $600 \AA$ and $912 \AA$. These lines cannot be observed in the Milky Way because interstellar hydrogen completely absorbs light at these wavelengths from any sources beyond a small region around the Sun. This is unfortunate because the FUV is rich in spectral diagnostics of physical conditions and gas composition (23). Indeed, these lines are 

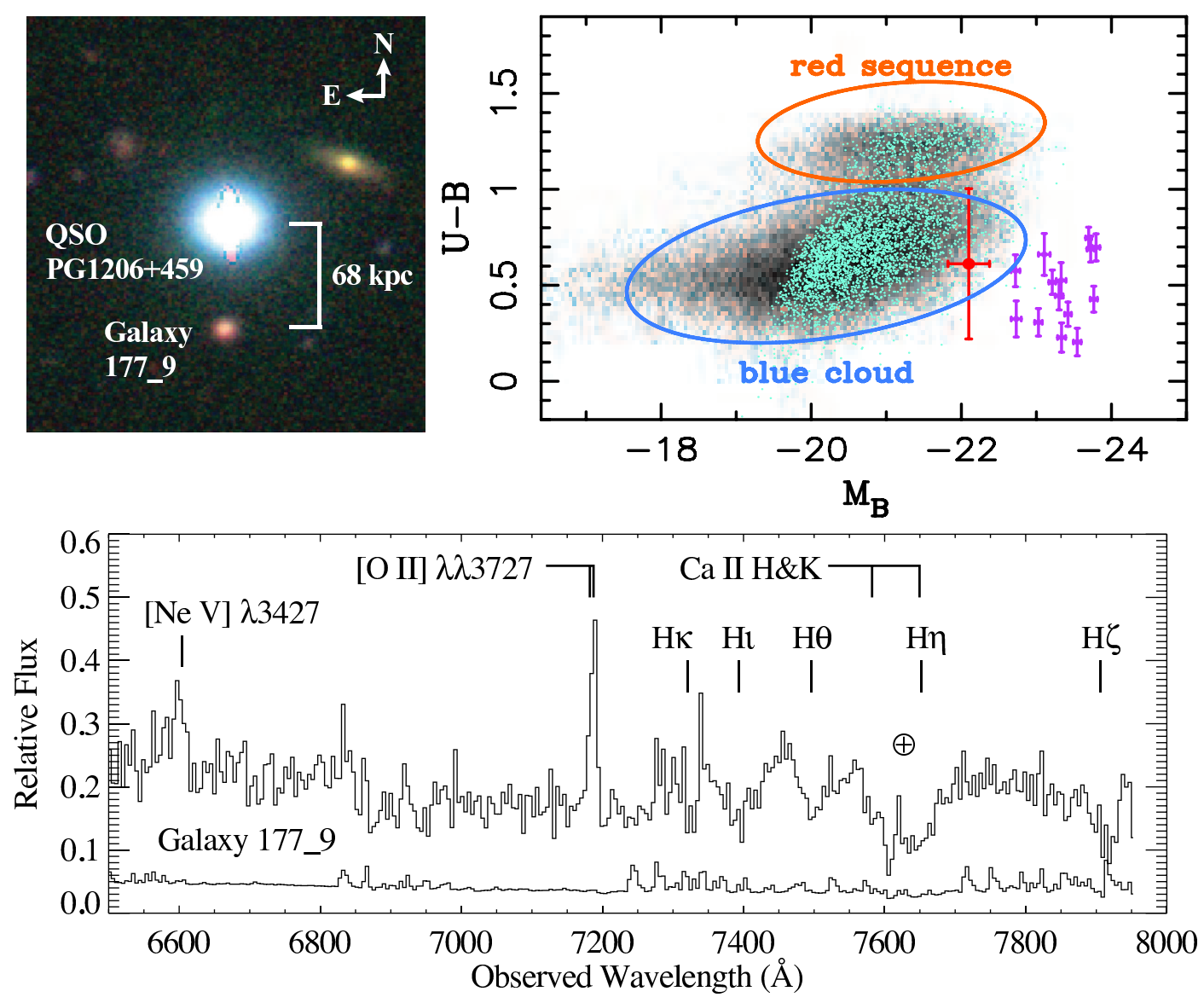

Figure 4: Montage of observations of the galaxy at $z_{\text {gal }}=0.927$ that drives a large-scale outflow of metal-enriched plasma. The galaxy, and the background QSO which reveals the outflow via absorption spectroscopy, is shown in the upper-left panel in a multicolor image obtained with the Large Binocular Telescope. This galaxy, which we refer to as 177_9, is the red object 8.63 arcseconds south of the bright QSO PG1206+459 $\left(z_{\mathrm{QSO}}=1.1625\right)$ at a position angle of $177^{\circ}$ ( $\mathrm{N}$ through $\mathrm{E}$ ) from the QSO. At the galaxy redshift, the angular separation from the QSO sight line corresponds to an impact parameter of $68 \mathrm{kpc}$. The large red circle in the upper-right panel indicates the rest-frame U-B color and absolute B magnitude of 177_9 compared to all galaxies from the DEEP2 survey (grayscale, 30) and DEEP2 galaxies within \pm 0.05 of $z$ (177_9) (cyan points). The small purple circles show poststarburst galaxies from (11). The lower panel shows an MMT optical spectrum of 177_9 (upper trace) with its $1 \sigma$ uncertainty (lower trace). The strong feature at $\approx 7600 \AA$ is partially due to telluric absorption. 
Table S1: Column Densities in the $z_{\mathrm{abs}}=0.927$ Absorption System of PG1206 +459

\begin{tabular}{|c|c|c|c|c|c|}
\hline Species & $\begin{array}{l}\text { Fitted } \\
\text { Transitions }(\AA)\end{array}$ & \multicolumn{4}{|c|}{$\begin{array}{c}\log \left[\text { Column Density }\left(\mathrm{cm}^{-2}\right)\right] \\
\text { (component velocity centroid, } \mathrm{km} \mathrm{s}^{-1} \text { ) }\end{array}$} \\
\hline \multicolumn{6}{|c|}{ Component Group A $\left(-450<v<0 \mathrm{~km} \mathrm{~s}^{-1}\right)$} \\
\hline & & $(-317)$ & $(-247)$ & $(-162)$ & $(-88)$ \\
\hline H I & $\operatorname{Ly} \alpha, \operatorname{Ly} \zeta, \operatorname{Ly} \eta$ & $14.96 \pm 0.11$ & $15.42 \pm 0.03$ & $15.39 \pm 0.04$ & $15.28 \pm 0.04$ \\
\hline $\mathrm{C}$ II & $687.05,1334.53$ & $13.65 \pm 0.03$ & $13.83 \pm 0.03$ & $13.80 \pm 0.05$ & $13.51 \pm 0.04$ \\
\hline $\mathrm{N}$ II & 644.63 & $12.71 \pm 0.25$ & $13.50 \pm 0.08$ & $13.09 \pm 0.13$ & $13.01 \pm 0.14$ \\
\hline Mg II & $2796.35,2803.53$ & $11.93 \pm 0.03$ & $12.47 \pm 0.02$ & $12.36 \pm 0.01$ & $12.16 \pm 0.02$ \\
\hline Si II & $1190.42,1193.29,1260.42,1526.71$ & $12.25 \pm 0.14$ & $12.24 \pm 0.17$ & $12.52 \pm 0.08$ & $12.43 \pm 0.11$ \\
\hline N III & $685.00,763.34$ & $14.50 \pm 0.02$ & $14.42 \pm 0.03$ & $14.20 \pm 0.04$ & $14.02 \pm 0.05$ \\
\hline O III & 702.33 & $\geq 14.65$ & $\geq 14.65$ & $\geq 14.52$ & $\geq 14.55$ \\
\hline S III & $698.73,724.29$ & $13.17 \pm 0.03$ & $13.21 \pm 0.03$ & $13.19 \pm 0.03$ & $12.88 \pm 0.06$ \\
\hline $\mathrm{N}$ IV & 765.15 & $\geq 14.24$ & $\geq 14.37$ & $\geq 14.14$ & $\geq 14.06$ \\
\hline O IV & 787.71 & $\geq 14.91$ & $\geq 14.88$ & $\geq 14.82$ & $\geq 14.86$ \\
\hline Si IV & $1393.76,1402.77$ & $13.00 \pm 0.04$ & $12.94 \pm 0.05$ & $13.05 \pm 0.09$ & $13.16 \pm 0.04$ \\
\hline S IV & 657.34 & $13.20 \pm 0.03$ & $13.25 \pm 0.03$ & (blended) & (blended) \\
\hline $\mathrm{N} \mathrm{V}$ & $1238.82,1242.80$ & $14.30 \pm 0.04$ & $14.30 \pm 0.05$ & $13.40 \pm 0.18$ & $13.96 \pm 0.08$ \\
\hline S V & 786.48 & $12.88 \pm 0.03$ & $12.84 \pm 0.03$ & $12.67 \pm 0.05$ & $12.31 \pm 0.08$ \\
\hline $\mathrm{Ne}$ VIII & $770.41,780.32$ & $13.71 \pm 0.29$ & $14.04 \pm 0.08$ & (not detected) & $14.07 \pm 0.04$ \\
\hline $\operatorname{Mg} X$ & 624.95 & $\longleftarrow<1$ & $04 \longrightarrow$ & $\longleftarrow<1$ & $94 \longrightarrow$ \\
\hline \multicolumn{6}{|c|}{ Component Group B $\left(0<v<300 \mathrm{~km} \mathrm{~s}^{-1}\right)$} \\
\hline & & $(65)$ & $(+108)$ & $(+159)$ & \\
\hline H I & $\operatorname{Ly} \alpha, \operatorname{Ly} \zeta, \operatorname{Ly} \eta, \operatorname{Ly} \nu$ & $16.28 \pm 0.13$ & $16.76 \pm 0.08$ & $16.32 \pm 0.07$ & \\
\hline $\mathrm{C}$ II & $687.05,1334.53$ & $\geq 14.10$ & $\geq 14.36$ & $13.88 \pm 0.07$ & \\
\hline N II & 644.63 & $14.01 \pm 0.04$ & $14.04 \pm 0.05$ & $13.62 \pm 0.12$ & \\
\hline Mg II & $2796.35,2803.53$ & $13.39 \pm 0.03$ & $13.36 \pm 0.01$ & $12.84 \pm 0.01$ & \\
\hline Si II & $1190.42,1193.29,1260.42,1526.71$ & $13.50 \pm 0.12$ & $13.39 \pm 0.04$ & $13.06 \pm 0.09$ & \\
\hline N III & 763.34 & $14.64 \pm 0.04$ & $14.54 \pm 0.13$ & $14.65 \pm 0.12$ & \\
\hline O III & 702.33 & (saturated) & (saturated) & (saturated) & \\
\hline S III & $698.73,724.29$ & $13.62 \pm 0.04$ & $13.58 \pm 0.04$ & $13.66 \pm 0.03$ & \\
\hline $\mathrm{N}$ IV & 765.15 & (saturated) & (saturated) & (saturated) & \\
\hline O IV & $608.40,787.71$ & (saturated) & (saturated) & (saturated) & \\
\hline Si IV & $1393.76,1402.77$ & $13.32 \pm 0.03$ & $13.28 \pm 0.04$ & $13.47 \pm 0.03$ & \\
\hline S IV & 657.34 & $13.47 \pm 0.04$ & $13.13 \pm 0.09$ & $13.66 \pm 0.04$ & \\
\hline $\mathrm{N} \mathrm{V}$ & $1238.82,1242.80$ & $13.93 \pm 0.06$ & $13.94 \pm 0.10$ & $13.84 \pm 0.11$ & \\
\hline $\mathrm{S}$ V & 786.48 & $12.93 \pm 0.04$ & (blended) & (blended) & \\
\hline $\mathrm{Ne}$ VIII & $770.41,780.32$ & $\longleftarrow \quad 14.53$ & $0.04 \longrightarrow$ & $14.21 \pm 0.05$ & \\
\hline $\operatorname{Mg} X$ & 624.95 & & $<14.20$ & & \\
\hline \multicolumn{6}{|c|}{ Component Group C $\left(1000<v<1250 \mathrm{~km} \mathrm{~s}^{-1}\right)$} \\
\hline & & $(+1084)$ & $(+1131)$ & & \\
\hline H I & $\operatorname{Ly} \alpha, \operatorname{Ly} \zeta, \operatorname{Ly} \iota, \operatorname{Ly} \xi$ & $15.02 \pm 0.16$ & $15.93 \pm 0.03$ & & \\
\hline C II & 687.05 & $12.92 \pm 0.14$ & $13.58 \pm 0.04$ & & \\
\hline $\mathrm{N}$ II & 644.63 & (blended) & (blended) & & \\
\hline Mg II & $2796.35,2803.53$ & $<11.37$ & $12.10 \pm 0.04$ & & \\
\hline Si II & $1190.42,1193.29,1260.42,1526.71$ & $<12.37$ & $12.53 \pm 0.12$ & & \\
\hline N III & $685.00,763.34$ & (blended) & (blended) & & \\
\hline O III & 702.33 & $13.48 \pm 0.09$ & $\geq 14.64$ & & \\
\hline S III & $698.73,724.29$ & $<12.33$ & $13.55 \pm 0.03$ & & \\
\hline $\mathrm{N}$ IV & 765.15 & $12.53 \pm 0.12$ & $13.59 \pm 0.02$ & & \\
\hline O IV & $608.40,787.71$ & $13.95 \pm 0.05$ & $\geq 15.02$ & & \\
\hline Si IV & $1393.76,1402.77$ & (not detected) & $13.63 \pm 0.05$ & & \\
\hline S IV & 657.34 & $12.18 \pm 0.35$ & $13.30 \pm 0.03$ & & \\
\hline $\mathrm{N} v$ & $1238.82,1242.80$ & $<13.49$ & $<13.56$ & & \\
\hline $\mathrm{S}$ V & 786.48 & $11.87 \pm 0.32$ & $13.03 \pm 0.03$ & & \\
\hline $\mathrm{Ne}$ VIII & $770.41,780.32$ & $13.30 \pm 0.27$ & $13.78 \pm 0.09$ & & \\
\hline $\operatorname{Mg~X}$ & 624.95 & $\longleftarrow<1$ & $08 \longrightarrow$ & & \\
\hline
\end{tabular}


rarely observed in any astrophysical context, although they are powerful tools for solar physics. To access these diagnostics, we use quasars to search for absorption lines at redshifts $z>0.5$ so that the FUV transitions are redshifted into the standard HST bandpass ( $\lambda_{\text {HST }}>1150 \AA$ ). For example, $z_{\text {abs }}>0.5$ redshifts the Ne VIII 770.41,780.32 $\AA$ doublet into the wavelength range observable with HST. In principle, these lines can be studied from the ground for sufficiently high $z_{\text {abs }}$ systems, but in practice this is extremely difficult because the "Ly $\alpha$ forest" imprints hundreds of closely spaced absorption lines on the spectrum at such high redshifts and makes detailed analysis of FUV metal lines very difficult. More importantly, high-redshift QSO sightlines have a high probability of encountering an optically thick Lyman limit absorber that completely absorbs the QSO flux in the rest-frame FUV range. Consequently, we have elected to study FUV absorption lines at $z_{\text {abs }}<1.5$, which cannot be observed from the ground but are in a redshift range where the Ly $\alpha$ forest is much thinner, blending is less common, and optically thick LL systems are rare (even in this redshift range, some blending with interloping lines from other redshifts occurs, as can be seen from Figure 2). Accordingly, we have targeted QSOs at $z_{\mathrm{QSO}} \approx 1-1.5$ in order to have a large total redshift path over which to search for the Ne VIII doublet and the other FUV lines. Spectrographs on the HST before COS and on the Far Ultraviolet Spectroscopic Explorer generally could not target QSOs at $z \gg 0.5$ because they are mostly too faint to observe with those instruments in practical exposure times. Consequently, the Ne VIII doublet has been only rarely detected (27), and some of the lines in Table S1 have never been seen in interstellar or intergalactic contexts.

To overcome the occasional blending problem, we use two strategies to measure column densities. First, we use as many transitions as possible to constrain a given species by simultaneously fitting Voigt profiles to all of the lines. For example, we simultaneously fit the S III lines at 698.73 and $724.29 \AA$ (in principle, we could also fit the S III line at $677.75 \AA$, but this line is redshifted into a region that is badly affected by $\mathrm{O}$ I emission from the Earth's exosphere, and at the time of this writing, the COS data reduction software could not exclude that contamination). In velocity ranges where one transition is affected by a strong blend, that velocity range is masked out in the problematic line but is still constrained by the other transitions used in the fit. Second, in some cases we can estimate the strength of the blended interloper and divide it out of the profile of interest. An example of this technique is shown in Figure S1. The upper panel of this figure compares the apparent column density profiles (28) of the Ne VIII 770.41,780.32 $\AA$ lines in the component at $v=+1131 \mathrm{~km} \mathrm{~s}^{-1}$. In this component, the Ne VIII $780.32 \AA$ line is blended with an $\mathrm{H} \mathrm{I} \mathrm{Ly} \gamma$ line at $z_{\mathrm{abs}}=0.55137$. Fortunately, the $\mathrm{H}$ I Ly $\beta$ and $\mathrm{Ly} \epsilon$ lines are also clearly detected at $z_{\mathrm{abs}}=0.55137$, and by fitting the $\operatorname{Ly} \beta$ and $\mathrm{Ly} \epsilon$ transitions, we can predict the strength of the $\mathrm{Ly} \gamma$ line and remove it from the blend with the Ne VIII $780.32 \AA$ profile. The Ly $\gamma$ line modeled in this way is shown with a smooth blue line in the upper panel of Figure S1, and the lower panel of Figure S1 shows the Ne VIII 780.32 A profile after the correction for the Ly $\gamma$ blend has been applied. In the lower panel the two Ne VIII apparent column density profiles are in excellent agreement. We have used similar modeling to correct for the $\mathrm{H}$ I Ly $\beta$ line at $z_{\text {abs }}=0.46435$, which is somewhat blended with the Ne VIII $780.32 \AA$ line in the component at $v=-317 \mathrm{~km} \mathrm{~s}^{-1}$. 


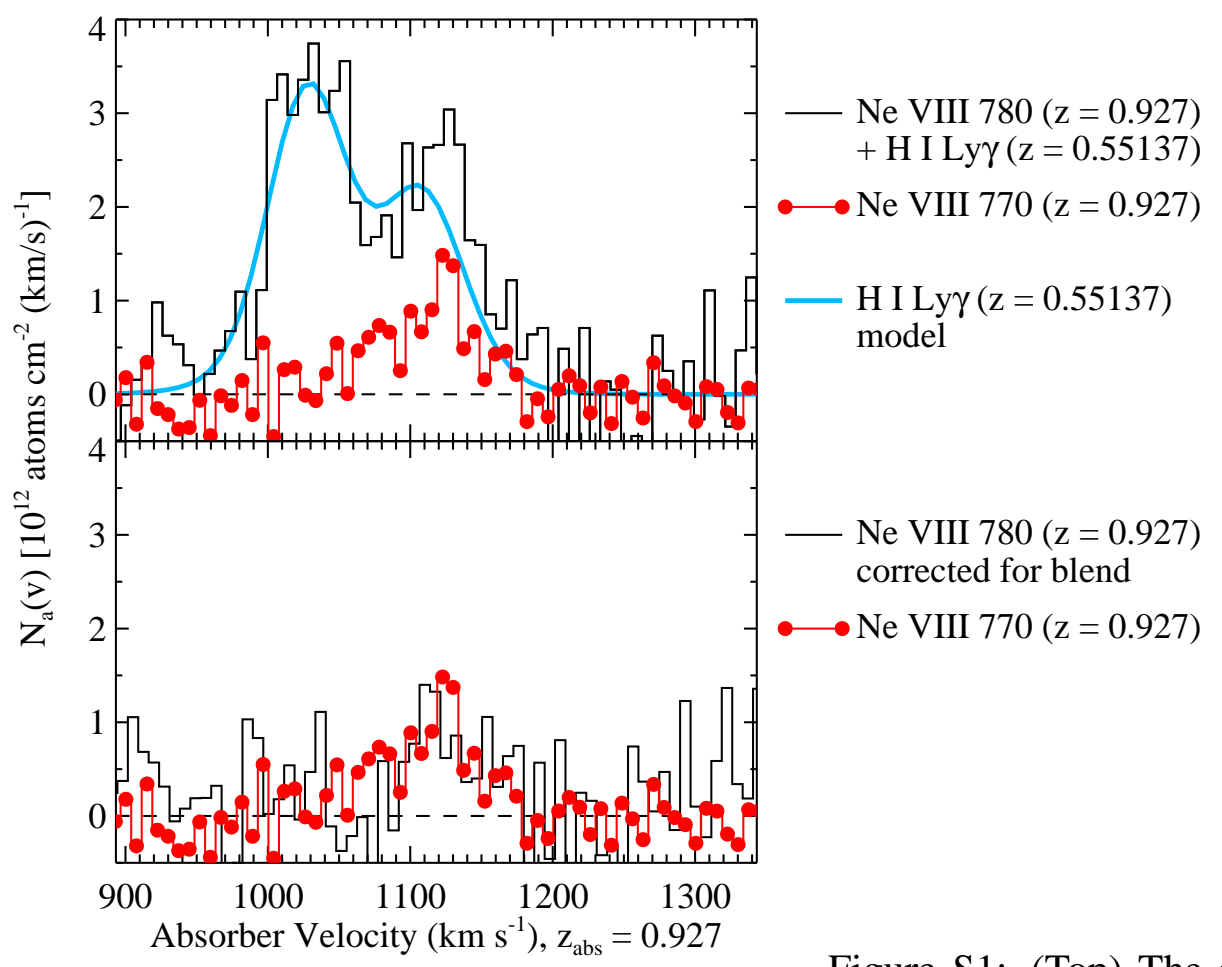

Figure S1: (Top) The apparent column density profiles (28) of Ne VIII $780 \AA$ (black lines) and Ne VIII $770 \AA$ (red lines with dots) before the $780 \AA$ line is corrected for a blend with $\mathrm{H} \mathrm{I} \mathrm{Ly} \gamma$ at $z_{\mathrm{abs}}=0.55137$. The smooth blue line shows a model of the Ly $\gamma$ line, based on the well-detected $\mathrm{H} \mathrm{I} \mathrm{Ly} \beta$ and Ly $\epsilon$ lines at the same redshift ( $\mathrm{Ly} \delta$ is lost in a strong blend). (Bottom) By correcting the data with the $\mathrm{H} \mathrm{I} \mathrm{Ly} \gamma$ model, the $\mathrm{H}$ I blend is effectively removed, and the residual profile of Ne VIII $780 \AA$ is found to be in excellent agreement with $770 \AA$. 

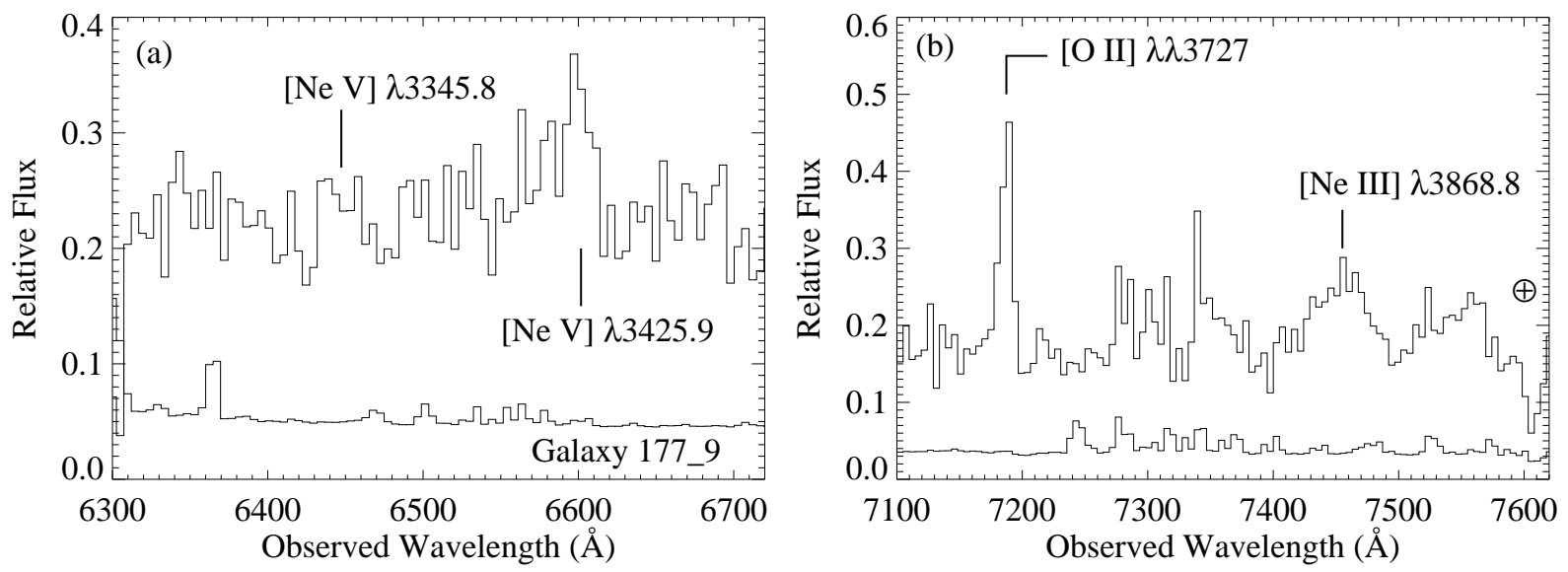

Figure S2: Expanded views of portions of the MMT spectrum of galaxy 177_9 from Figure 4 including (a) the region of the spectrum near the [Ne V] lines at 3345.8 and $3425.9 \AA$, and (b) the region near the [O II] emission at $3727 \AA$ and [Ne III] emission at $3868.8 \AA$. As in Figure 4, the upper histogram shows the flux from the galaxy and the lower histogram shows the $1 \sigma$ flux uncertainty.

With this approach, we have measured the column densities of a wide array of low- , intermediate- , and high-ionization metals in the PG1206 +459 absorption system at $z_{\text {abs }}=0.927$, and our column density measurements are listed in Table S1. For convenience in comparisons with previous work (24), we divide the components into the three groups they defined (groups $\mathrm{A}, \mathrm{B}$, and $\mathrm{C}$ ). We fit nine components, the maximum number we can effectively constrain with free fits to the COS data (four components in group A, three in group B, and two in group C). In some cases, the lines are so strong that saturation precludes a useful column density measurement, and in these cases we simply indicate that the component is "saturated" in Table S1. In other cases, the lines are strong enough to be moderately saturated but at a level that can be inferred from the profile shapes, especially when multiple lines of varying strength are fitted simultaneously. In these cases we report the column density indicated by profile fitting in Table S1, but we indicate the line as a lower limit because the profile fitting may still suffer significant systematic uncertainty due to saturation.

Properties of the affiliated galaxy. The MMT spectrum of galaxy 177_9 (Figure 4), shows highly significant [O II] emission along with a $4 \sigma$ emission feature that we identify as the [Ne V] $3426 \AA$ transition. This [Ne V] 3426 emission is often a signature of an active galactic nucleus (41); however, it is expected to be accompanied by weaker [Ne V] and [Ne III] emission features at 3346 and $3869 \AA$ (41). These weaker emission features are not immediately obvious in Figure 4, which calls the [Ne v] 3426 identification into question. However, as we show in Figure S2, the weaker neon emission lines are confused by ambiguous continuum placement 
as well as the intrinsic noisiness of the spectrum. A weak feature is evident at the expected wavelength of [Ne V] $\lambda 3346$ (Figure S2a). The significance of this feature depends on where the galaxy continuum is drawn in, but it is expected to be a factor of $\sim 3$ weaker than [Ne V] $\lambda 3426$ and thus is consistent with the stronger [Ne v] line. The [Ne III] $\lambda 3869$ line (Figure S2b) is nested between absorption lines and is close to the $4000 \AA$ break as well as a strong telluric absorption feature. The complexity of this spectral region, combined with noise, makes the continuum placement highly uncertain, and a [Ne III] $\lambda 3869$ feature with the expected strength could easily be present. Given the good agreement of the [Ne V] $\lambda 3426$ and [O II] $\lambda 3727$ features, we conclude that the [Ne v] $\lambda 3426$ identification is reliable. In turn, this implies that the [O II] $\lambda 3727$ emission might not be a suitable indicator of the star formation rate in 177_9 because the [O II] could be predominantly due to the AGN emission-line region $(42,43)$.

While it is interesting that the spectral features, high luminosity, and blue rest-frame color of galaxy 177_9 are similar to those of poststarburst galaxies $(11,37)$, we caution that its color and spectral features are noisy, and it is necessary to reduce these uncertainties to reliably classify the galaxy. The galaxy color is uncertain because it is derived from the Sloan Digital Sky Survey (SDSS) photometry of this object, and the galaxy is close to the SDSS detection limit. Unfortunately, the LBT images were not obtained in conditions suitable for photometry. For comparison with the DEEP2 galaxy color-magnitude diagram (Figure 4), we have transformed the SDSS photometry of 177_9 into a rest-frame U-B color using the K-correction and methods of (29), and this introduces some systematic uncertainty. However, the poststarburst galaxies from (11) shown in Figure 4 were transformed from SDSS filters to rest-frame U-B in an identical fashion, and the offset of these poststarburst galaxies from the "blue cloud" of ordinary galaxies is similar to the offset seen in SDSS filters directly (37). Deeper photometry of 177_9 would help to elucidate its nature.

We also note that another bright galaxy, apart from 177_9, is evident close to the PG1206+459 sight line (the yellow object northwest of the QSO in Fig. 4). We obtained an MMT spectrum of this galaxy, but unfortunately the spectrum does not reliably constrain its redshift. However, the absence of features in the wavelength range of the MMT spectrum, the angular extent of this galaxy, and its color suggest that it has $z \ll 0.927$. This conclusion is corroborated by the photometric redshifts of this object from the SDSS database, which range from $z_{\text {phot }}=0.21$ (44) to $z_{\text {phot }}=0.43(45)$.

Ionization, metallicity, and mass: cool phase. We obtain accurate $\mathrm{H}$ I measurements from the Lyman series lines (Figs. 1-2). The H I column densities are not high enough to prevent photoionization by the UV background light. Consequently, to estimate the total mass we apply standard photoionization models to account for the ionized hydrogen (H II) using CLOUDY, v8.0 (33). We assume the gas is photoionized by the diffuse UV background from quasars (46) and require the models to match the observed $N(\mathrm{H} \mathrm{I}), N(\mathrm{~S}$ III), and $N(\mathrm{~S}$ IV) on a componentby-component basis. We prefer S III and S IV because sulfur does not deplete onto dust and the lines are weak enough so that they are not confused by unresolved saturation. Some components are blended with unrelated lines from other redshifts, but we account for blends (and in some 
cases remove them) by employing multiple transitions (see above). We characterize the models by the ionization parameter (三 ionizing photon density/particle density), which is adjusted to fit the S III/S IV ratio, and then the metallicity is adjusted to fit the observed column densities with $N(\mathrm{H} \mathrm{I})$ fixed to the observed value. We have modeled the components at $v=-317,-247,65$, and $1131 \mathrm{~km} \mathrm{~s}^{-1}$ (the components with good S III/S IV measurements); examples of the models and results are shown in Figure S3. We do not expect these models to fit perfectly - there are many uncertain model parameters such as the shape and intensity of the UV background, the relative abundance patterns (we assume the solar pattern), and confusion due to dust depletion. Given these caveats, the models (Figure S3) compare reasonably well to the observations with the ionization parameter fixed by S III/S IV, most of the remaining low- and intermediateionization stages agree with the models to within $0.3-0.5$ dex. We obtain several robust results: First, while the metallicity is uncertain by $\approx 0.3$ dex due to radiation field uncertainties, the gas must be highly enriched (Table S2). Second, the observed Ne VIII, N V, and S V columns are significantly higher (in some cases, by orders of magnitude) than predicted by the photoionization models. These species must arise in a separate, higher ionization phase. Third, the models require high total hydrogen $(\mathrm{H} \mathrm{I}+\mathrm{H}$ II) columns even with high metallicities, and this in turn implies large masses.

We can obtain a rough estimate of the mass $(M)$ in the outflowing components using a standard thin-shell model (47): $M=\mu m_{p} N_{\perp}(\mathrm{H})_{\text {total }} \Omega r^{2}$, where $r$ is the shell radius ( $\geq$ impact parameter $=68 \mathrm{kpc}), N_{\perp}(\mathrm{H})_{\text {total }}$ is the total $\mathrm{H}$ column perpendicular to the shell surface, $\Omega$ is the shell solid angle, $m_{p}$ is the proton mass, and $\mu=1.3$ accounts for the additional mass of helium. To calculate $\Omega$, we assume the outflow full opening angle $\theta$ ranges between 45 and $135^{\circ}$ as observed in nearby starburst and AGN outflows (6). Unlike many previous studies, we are not observing a sight line directly toward the galaxy that launches the outflow, so me must convert our observed total hydrogen column densities (along the line of sight) into $N_{\perp}(\mathrm{H})_{\text {total }}$. To make this conversion, we adopt the simplified geometry shown in Figure S4. We detect absorption at both negative and positive velocities with respect to the galaxy (Figure 2), and despite the large velocity separation, the positive- and negative-velocity gas has similar properties. This can be understood if we are detecting the front and back sides of a biconical outflow, as illustrated in Figure S4, but this requires that the sight line is roughly parallel to the outflow axis (or else only the front or back side shell would be detected, but not both). In this situation, if the sight line to the background QSO is at large enough impact parameter $(\rho)$, it will not penetrate the shells and no absorption will be detected (position A in Figure S4). At the maximum impact parameter that intercepts the shells (position B), we see that $r=\rho / \sin (\theta / 2)$ and $N_{\perp}(\mathrm{H})=N(\mathrm{H})_{\text {total }} \cos$ $(\theta / 2)$. As the sight line is moved to smaller impact parameters (e.g., position $\mathrm{C}$ ), $r$ will increase and $N(\mathrm{H})_{\text {total }} \longrightarrow N_{\perp}(\mathrm{H})$, both of which will increase the mass compared the value calculated assuming the sight line is at position B. Thus, by calculating the mass assuming the sight line is near position $\mathrm{B}$, we derive a lower limit. With these assumptions, we obtain the $N_{\perp}(\mathrm{H})$ values and masses summarized in Table $\mathrm{S} 2$ for the cool (low-ionization) phase.

Of course, this model is highly simplified compared to the complex situation that is likely in real outflows. For example, the schematic sight lines in Figure S4 could be tilted toward the 


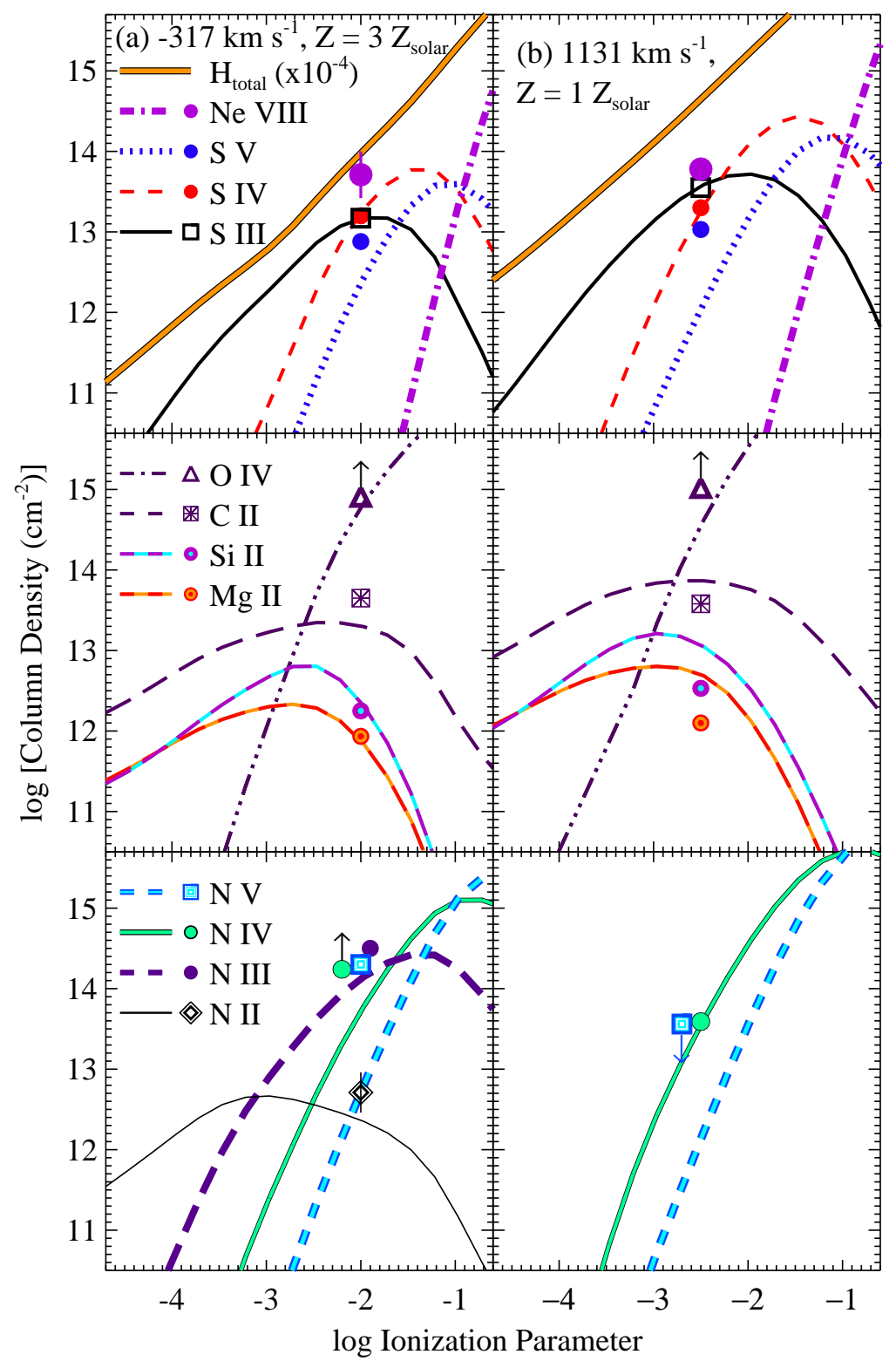

Figure S3: Comparison of the observed column densities in two of the absorber components to predictions from a CLOUDY (33) photoionization model. The columns show results for the components at (a) $-317 \mathrm{~km} \mathrm{~s}^{-1}$, and (b) $+1131 \mathrm{~km} \mathrm{~s}^{-1}$. In each panel, the column densities predicted by the model are shown with smooth curves and the observed columns are plotted with discrete symbols at the ionization parameter that provides the best fit to the observed S III and S IV column densities. Some species (e.g., N IV, N v, S v, Ne VIII) do not agree with the models due to contributions from the hot-gas phase (Figure S5). The species corresponding to the curves and discrete symbols are indicated by the legends in column (a). Column density uncertainties are usually smaller than the symbol sizes. The underabundance of Mg II and Si II in (b) could be due to dust, which depletes these species, but these ions are sensitive to the shape of the ionizing flux field. 


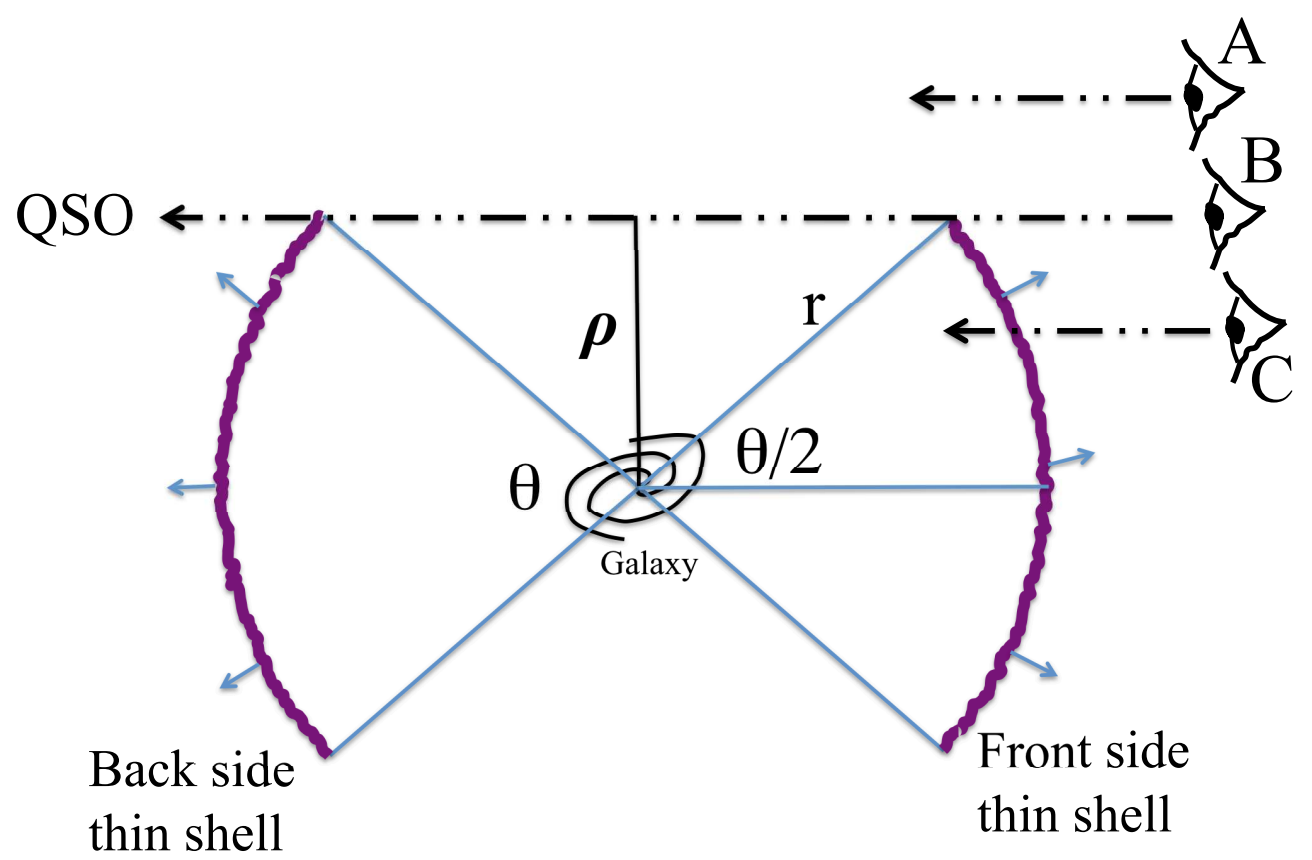

Figure S4:

Schematic sketch of the simplified geometry assumed to roughly estimate shell mass. We assume that the sight line to the background QSO is roughly parallel to the outflow axis so that the front and back sides of the biconical outflow are intercepted by the sight line. We assume that the shells are at the cone caps, so an observer at position A would not detect the outflow because that sight line does not intercept the bicone. Position $\mathrm{B}$ represents the maximum impact parameter that pierces the shell, and at this position, $N_{\perp}(\mathrm{H})=N(\mathrm{H})_{\text {total }} \cos (\theta / 2)$, where $\theta$ is the full opening angle. As the observer moves from position $\mathrm{B}$ toward position $\mathrm{C}$ and toward viewing the galaxy directly, $N(\mathrm{H})_{\text {total }} \longrightarrow N_{\perp}(\mathrm{H})$, and $r$ increases, both of which increase the calculated mass in the shell. Therefore, the mass calculated assuming position B provides a lower limit on the true mass.

Table S2: Metallicities (Elemental Abundances) and Thin-Shell Masses of Individual Components in the Absorption Line System

\begin{tabular}{llccccccc}
\hline \hline \multirow{2}{*}{$\begin{array}{l}\text { Component } \\
\text { Velocity } \\
\left(\mathrm{km} \mathrm{s}^{-1}\right)\end{array}$} & Metallicity & $\log N(\mathrm{H})_{\text {total }}$ & $\log N_{\perp}(\mathrm{H})_{\text {total }}$ & $\begin{array}{c}\text { Mass } \\
\left(10^{8} M_{\odot}\right)\end{array}$ & $\log N(\mathrm{H})_{\text {total }}$ & $\log N_{\perp}(\mathrm{H})_{\text {total }}$ & $\begin{array}{c}\text { Mass } \\
\left(10^{8} M_{\odot}\right)\end{array}$ & $\begin{array}{c}\text { Temperature } \\
\left(10^{5} \mathrm{~K}\right)\end{array}$ \\
\hline-317 & & & & & & & \multicolumn{2}{c}{ Hot Phase } \\
-247 & $1 Z_{\text {solar }}$ & 18.0 & $17.6-18.0$ & $0.6-1$ & $19.2-19.8$ & $18.8-19.8$ & $10-80$ & $2.2-3.8$ \\
+65 & $1 Z_{\text {solar }}$ & 18.5 & $18.1-18.5$ & $2-4$ & $20.1-20.4$ & $19.7-20.3$ & $80-300$ & $3.3-4.0$ \\
+1131 & $0.5 Z_{\text {solar }}$ & 19.1 & $18.7-19.1$ & $8-14$ & $20.4-20.5$ & $19.9-20.5$ & $100-400$ & $4.2-4.5$ \\
\hline
\end{tabular}


galaxy somewhat, and front- and back-side absorption would still be detected. However, the sight line must have an impact parameter of $68 \mathrm{kpc}$, so as it is tilted toward the galaxy, the radius becomes larger and $N(\mathrm{H})_{\text {total }} \longrightarrow N_{\perp}(\mathrm{H})$, so our mass calculations are still lower limits in this situation. More importantly, the geometry of the outflowing material could be completely different from thin shells. If the absorption arises in a context similar to the multiple bubbles and filaments seen near some AGN in the nearby universe $(48,49)$ or the multifilament outflows extending away from the disks of nearby starburst galaxies (6), then the geometry is much more complex, although the absorption could still originate in a thin shell or filament. In filaments, the mass could be substantially lower. For example, the optical filaments of NGC1275 are long and skinny with dimensions of $0.07 \mathrm{kpc}$ by $6 \mathrm{kpc}(35)$ and thus have a cold-gas mass $\approx 10^{6} M_{\odot}$. However, as discussed below, the total column density and mass in the Ne VIII phase is substantially higher than the cold-gas mass. The network of optical filaments centered on NGC1275 extend up to $\approx 70 \mathrm{kpc}$ from the galaxy (50), so the scale of NGC1275 emission is similar to the scale of the absorption near galaxy 177_9. The complex velocity distribution of the absorption lines near 177_9 (Figure 1 - 2) - with more components at negative velocities than positive velocities - could arise naturally in a set of bubbles, shells, and filaments like those surrounding NGC1275. We note that magnetic fields have been suggested to stabilize the skinny filaments of NGC1275 (35) as well as many other central cluster galaxies (51) and starburst galaxies (6). It would be interesting to consider whether magnetically confined gas could prevent the low- and high-ionization gas from developing different kinematics and thus explain the striking similarity and alignment of the low- and high-ionization lines in the PG1206+459 absorber (Figure 3).

At this time, many configurations and models remain viable for the absorption affiliated with 177_9, so the thin-shell masses in Table S2 should be viewed as order-of-magnitude calculations, but it is interesting to note that even though there are large uncertainties, the absorbing gas is likely to contain substantial mass. It is also possible to consider thick shells and clumping of the gas within the shells (47), but more detailed observational constraints must be obtained for such models.

Ionization and mass: hot phase. We see from Fig. S3 that the observed N V and Ne VIII must arise from a more highly ionized and hotter phase than the low ions. The strong correlation between the velocity centroids and profile shapes of the low- and high-ions suggest a physical connection; the highly ionized species may arise in the evaporating/ablating surface of the outflowing cool clouds or, conversely, the cool clouds may be rapidly cooling and condensing out of the hot outflow. Some of the intermediate ions (N IV, O IV, S V) may exist in both the photoionized and hot phases. To estimate the mass in the highly ionized phase in light of these possibilities, we use the Ne VIII/N V ratio to constrain the hot-phase gas temperature using three collisional ionization models (52), including rapidly cooling models, as shown in Figure S5. Then we assume the Ne VIII phase has the same metallicity as the cooler (photoionized) cloud at the same velocity and adjust $N(\mathrm{H})_{\text {total }}$ to match the observed $N(\mathrm{Ne}$ VIII) and $N(\mathrm{~N} v)$. Figure S5 shows examples of the models for the -317 and $-247 \mathrm{~km} \mathrm{~s}^{-1}$ components, including the isochoric and isobaric nonequilibrium models and the collisional ionization equi- 


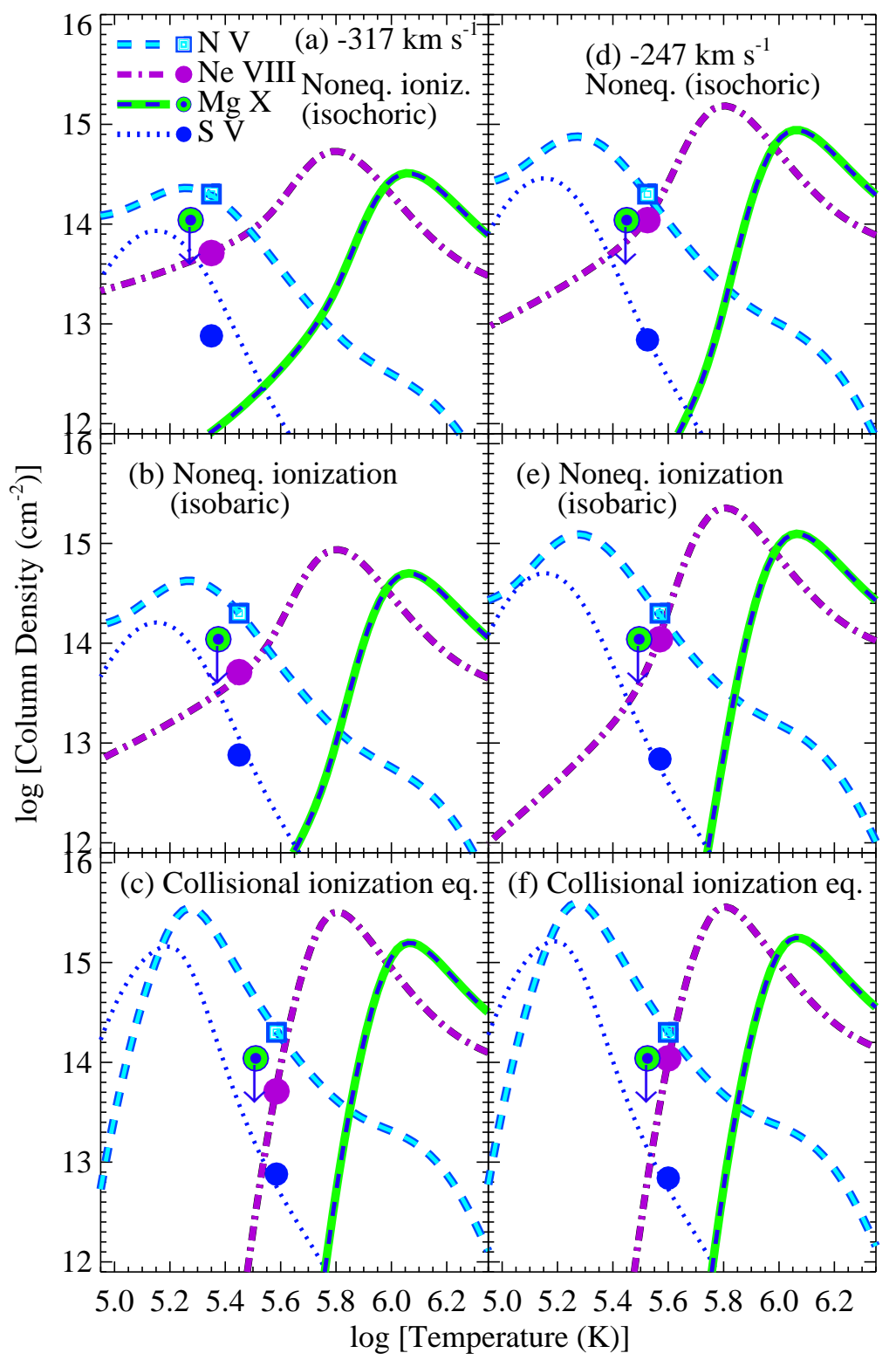

Figure S5: (a) Comparison of the observed $\mathrm{N} \mathrm{v}, \mathrm{S} \mathrm{V}$, and $\mathrm{Ne}$ VIII column densities to predictions from equilibrium and nonequilibrium collisional ionization models. Observed columns are plotted with discrete symbols at the temperature that provides the best fit to the models, which are shown with smooth curves. Species corresponding to the symbols and curves are indicated in the legend at upper left. The left column shows fits for the component at $v=-317 \mathrm{~km} \mathrm{~s}^{-1}$ for the following models (52): (a) nonequilibrium ionization in plasma that is rapidly and isochorically cooling (i.e., at constant density) from an initial temperature of $5 \times 10^{6} \mathrm{~K}$, (b) same but for isobaric (constant pressure) nonequilibrium cooling gas, and (c) collisional ionization equilibrium. Panels (d) (f) show the same models for the component at $v=-247 \mathrm{~km} \mathrm{~s}^{-1}$. In both components, $\mathrm{Mg} \mathrm{X}$ is not detected, and a $3 \sigma$ upper limit is shown. 
librium (CIE) model from (52). Table S2 summarizes the masses (from the thin-shell model above) and gas temperatures derived for the hot phase of three of the components; the lowest masses and temperatures in Table $\mathrm{S} 2$ result from the isochoric nonequilibrium (rapidly cooling) model, and the highest masses/temperatures result from assuming CIE. We find that all three models provide acceptable fits and imply comparable masses. Interestingly, when these models are fit based on Ne VIII/N v, they naturally predict about the right amount of $\mathrm{S} \mathrm{V}$. The models are also consistent with the upper limits on $\mathrm{Mg} \mathrm{X}$ and the looser constraints on species that are more uncertain due to moderate saturation (e.g., N IV, O IV). We have also investigated whether the Ne VIII phase could arise in gas photoionized by AGN flux from the affiliated galaxy. This model does not work well - we cannot match the Ne VIII/N v without significantly violating other constraints (other column densities) from the data.

In principle, the hot gas could have a higher metallicity than the cool gas. The hot extraplanar gas of NGC1569, for example, has a higher metallicity than its underlying H I disk (53), which suggests that chemically enriched hot gas is preferentially escaping into the halo, as predicted in some galactic wind models (54). However, the high degree of similarity of the lowand high-ion profiles in the PG1206+459 absorber (Figure 3) argues against this situation. If the cool and hot gas had separated sufficiently so that the hot phase had significantly higher metallicity, then the cool and hot gas would have different kinematical properties. Instead, the lowand high-ion profiles are remarkably similar. We note that in the central galaxies of coolingflow clusters, the metallicity of the hot phase has been estimated to range from $1 \times$ to $3 \times$ the metallicity of the cooler phase based single-temperature models fit to X-ray emission (51), so our assumption of similar metallicites in the cool and hot phases would be reasonable in this context. However, as emphasized in (51), there are substantial systematic uncertainties in those metallicity estimates, and in some cases the signal-to-noise is not adequate to support definitive conclusions. Moreover, the "cool" phase metallicity is also based on X-ray emission data with low spectral resolution, so we do not know if the cool and hot phases in that study are as well-aligned kinematically as the UV data presented in this paper.

\section{References and Notes}

1. P. F. Hopkins et al., Astrophys.J.Suppl.Ser. 163, 1 (2006)

2. A. I. Zabludoff et al., Astroph.J., 466, 104 (1996)

3. G. F. Snyder, T. J. Cox, C. C. Hayward, L. Hernquist, P. Jonsson, Astroph.J., 741, 77 (2011)

4. D. Kereš, N. Katz, R. Davé, M. Fardal, D. H. Weinberg, Mon.Not.R.Astron.Soc., 396, 2332 (2009)

5. B. D. Oppenheimer et al., Mon.Not.R.Astron.Soc., 406, 2325 (2010)

6. S. Veilleux, G. Cecil, J. Bland-Hawthorn, Ann. Rev. Astron. Astrophys., 43, 769 (2005) 
7. T. M. Heckman, M. D. Lehnert, D. K. Strickland, L. Armus, Astrophys.J.Suppl.Ser., 129, 493 (2000)

8. D. S. Rupke, S. Veilleux, D. B. Sanders, Astrophys.J.Suppl.Ser., 160, 87 (2005)

9. C. L. Martin, Astroph.J., 647, 222 (2006)

10. M. Pettini et al. Astroph.J., 554, 981 (2001)

11. C. A. Tremonti, J. Moustakas, A. M. Diamond-Stanic, Astroph.J., 663, L77 (2007)

12. C. C. Steidel et al., Astroph.J., 717, 289 (2010)

13. K. H. R. Rubin et al., Astroph.J., 719, 1503 (2010)

14. F. Hamann, G. Ferland, Ann. Rev. Astron. Astrophys., 37, 487 (1999)

15. J. P. Grimes et al., Astrophys.J.Suppl.Ser., 181, 272

16. K. H. R. Rubin et al., Astroph.J., 712, 574 (2010)

17. M. Moe, N. Arav, M. A. Bautista, K. T. Korista, Astroph.J., 706, 525 (2009)

18. J. P. Dunn et al., Astroph.J., 709, 611 (2010)

19. D. Edmonds et al., Astroph.J., 7397 (2011)

20. F. Hamann et al., Mon.Not.R.Astron.Soc., 410, 1957 (2011)

21. G. A. Kriss et al., Astro. \& Astroph., 534, 41 (2011)

22. D. M. Capellupo, F. Hamann, J. C. Shields, P. Rodríguez Hidalgo, T. Barlow, Mon.Not.R.Astron.Soc., 413, 908 (2011)

23. D. A. Verner, D. Tytler, P. D. Barthel, Astroph.J., 430, 186 (1994)

24. J. Ding, J. C. Charlton, C. W. Churchill, C. Palma, Astroph.J., 590, 746 (2003)

25. See further information in supporting material in Science online.

26. B. D. Savage, N. Lehner, B. P. Wakker, K. R. Sembach, T. M. Tripp, Astroph.J., 626, 776

27. A. Narayanan et al., Astroph.J., 730, 15 (2011)

28. Y. Chen, J. D. Lowenthal, M. S. Yun, Astroph.J., 712, 1385 (2010)

29. The galaxy appears to be red in Fig. 4 due to its redshift; in the rest frame of the galaxy, it has a very blue U-B color. 
30. C. N. A. Willmer et al., Astroph.J., 647, 853 (2006)

31. For distance calculations, we assume a cold dark matter cosmology with $H_{0}=70 \mathrm{~km} \mathrm{~s}^{-1}$ $\mathrm{Mpc}^{-1}, \Omega_{\mathrm{m}}=0.30, \Omega_{\Lambda}=0.70$.

32. G. J. Ferland et al., Pub.Astr.Soc.Pac., 110, 761. (1998)

33. As discussed in the supporting material, the yellow galaxy northwest of the QSO (Figure 4) does not have a spectroscopic redshift but is likely to have $z \ll 0.927$.

34. A. C. Fabian et al., Nature, 454, 968 (2008)

35. T. M. Tripp et al., Astroph.J.Supp.Ser., 177, 39 (2008)

36. C. Tremonti, A. M. Diamond-Stanic, J. Moustakas, ASP Conf. Ser., 419, 369 (2009)

37. A. L. Coil et al. 2011, http://arXiv.org/abs/1104.0681(2011)

38. J. Tumlinson et al., Science, 334, 948 (2011)

39. B. D. Savage, K. R. Sembach, Astroph.J., 379, 245 (1991)

40. J. D. Meiring et al., Astroph.J., 732, 35 (2011)

41. G. A. Shields, R. R. Ludwig, S. Salviander, Astroph.J., 721, 1835 (2010)

42. N. P. Abel, S. Satyapal, Astroph.J., 678, 686 (2008)

43. R. Yan et al., Astroph.J., 648, 281 (2006)

44. I. Csabai et al., Astron.J., 125, 580 (2003)

45. H. Oyaizu et al., Astroph.J., 674, 768 (2008)

46. F. Haardt, P. Madau, http://arXiv.org/abs/1105.2039 (2011)

47. D. S. Rupke, S. Veilleux, D. B. Sanders, Astrophys.J.Suppl.Ser., 160, 115 (2005)

48. E. L. Blanton, C. L. Sarazin, B. R. McNamara, Astrophys.J., 125, 1635 (2003)

49. A. C. Fabian et al., http://arXiv.org/abs/1105.5025 (2011)

50. C. J. Conselice, J. S. Gallagher, R. F. G. Wyse, Astron.J., 122, 2281 (2001)

51. M. McDonald, S. Veilleux, D. S. N. Rupke, R. Mushotzky, Astrophys.J., 721, 1262

52. O. Gnat, A. Sternberg, Astrophys.J.Suppl.Ser., 168, 213 (2007)

53. C. L. Martin, H. A. Kobulnicky, T. M. Heckman, Astrophys.J., 574, 663 (2002) 
54. M.-M. Mac Low, A. Ferrara, Astrophys.J., 513, 142 (1999)

Acknowledgements Based on observations made with the NASA/ESA Hubble Space Telescope, the MMT, a joint facility operated by the Smithsonian Astrophysical Observatory and the University of Arizona, and the LBT, an international collaboration among institutions in the United States, Italy, and Germany. Support for HST Program number 11741 was provided by NASA through a grant from the Space Telescope Science Institute, which is operated by the Association of Universities for Research in Astronomy, Incorporated, under NASA contract NAS5-26555. Additional support was provided by NASA grant NNX08AJ44G. The DEEP2 survey was supported was by NSF grants AST 95-29098, 00-711098, 05-07483, 08-08133, 00-71048, 05-07428, and 08-07630. Funding for SDSS has been provided by the Alfred P. Sloan Foundation, the Participating Institutions, the National Aeronautics and Space Administration,the National Science Foundation, the U.S. Department of Energy Office of Science, the Japanese Monbukagakusho, and the Max Planck Society. We thank Chris Churchill for providing the archival Keck data and the referees for review comments that significantly improved this paper. We are also grateful to the Hawaiian people for graciously allowing us to conduct observations from Mauna Kea, a revered place in the culture of Hawaii. The Hubble Space Telescope data in this paper is available from the MAST archive at http://archive.stsci.edu. 